\title{
KRAS NM_004985.3:c.34_36delGGTinsTGG
}

National Cancer Institute

\section{Source}

National Cancer Institute. KRAS NM 004985.3:C.34 36delGGTinsTGG. NCI Thesaurus.

Code C98395.

A complex substitution where the nucleotide sequence at positions 34 through 36 of the coding sequence of the KRAS gene has changed from guanine-guanine-thymine to thymine-guanine-guanine. 